\title{
IMPROVING FAULT DETECTION BY EXTENDED KALMAN FILTER ADJUSTMENT FOR OSCILLATORY FAILURE CASE IN AIRCRAFTS
}

\author{
D. Efimov, A. Zolghadri, and P. Simon \\ Automatic Control Group \\ IMS-lab, University of Bordeaux \\ 351 Cours de la Libération, Talence 33405, France
}

\begin{abstract}
The problem of model-based fault detection is studied with application of the Kalman filter for residual generation. The filter has two important incoming parameters, the state noise and the output noise covariance matrices, which tuning is analyzed in order to optimize the fault detection performance. The problem is formulated through an appropriate optimization criteria and applied to oscillatory failure case detection in aircraft control surfaces. The results of simulation illustrate efficiency of the proposed technique.
\end{abstract}

\section{INTRODUCTION}

The international airworthiness regulations applied worldwide by all aircraft manufactures and used for aircraft certification restrict admissible hazardous loads on the aircraft [1]. Some failures of the electronic flight control system may result in an unwanted control surface oscillation, generating unacceptably high loads or vibrations on the aircraft structure. This failure is called Oscillatory Failure Case (OFC) [1]. The capability to detect these failures is very important because it has an impact on the structural design of the aircraft. By proper design, the OFC amplitude must be maintained within an envelope function of the frequency. Usual monitoring techniques cannot always guarantee safeguarding within an envelope with acceptable robustness; thus, a specific OFC detection algorithm has to be designed [1].

In this paper, only OFC located in the servocontrol loop of the moving surfaces is considered (Fig. 1) [1]. Habitually, OFC generates spurious sinusoidal 


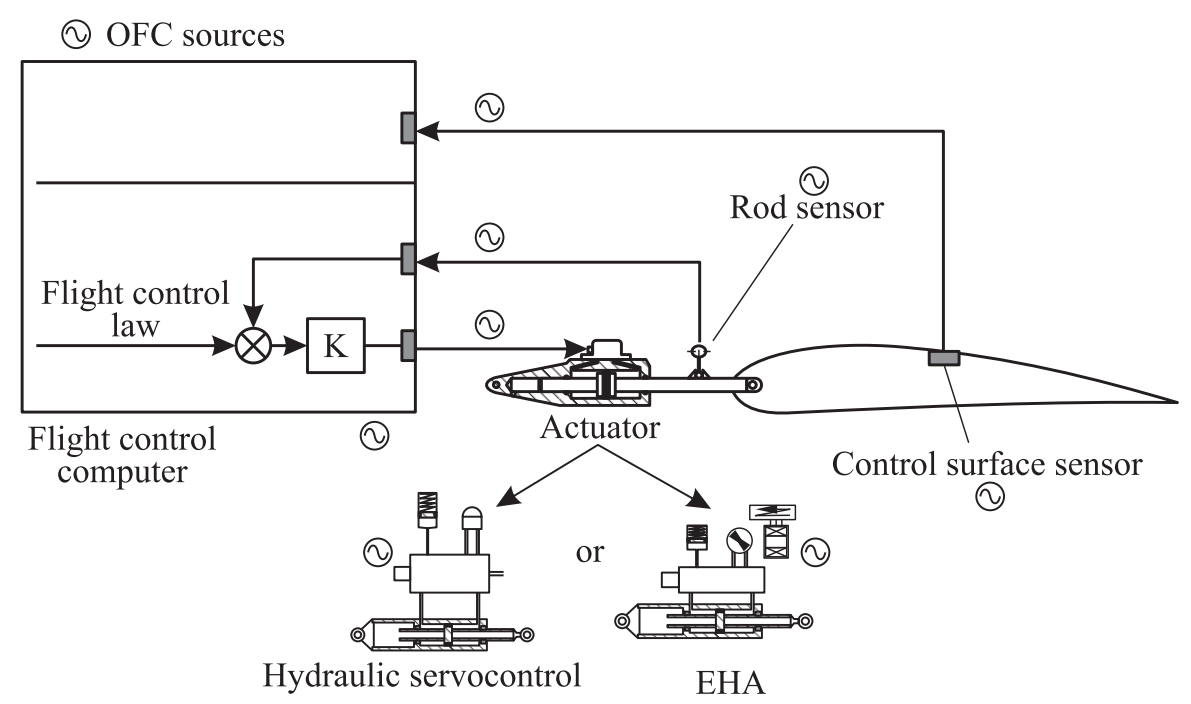

Figure 1 An OFC source location in the control loop

signals (mainly, due to electronic components) propagating through the servocontrol loop, leading to control surface oscillation. The faulty components are located inside the flight control computer, the analogue inputs/outputs, the position sensors, or the actuators. The flight control computer may also generate unwanted oscillations of the command current sent to the actuator servovalve. The OFC signals are considered as sinusoidal signals with amplitude and frequency uniformly distributed over the range $0-10 \mathrm{~Hz}$ (above $10 \mathrm{~Hz}, \mathrm{OFC}$ has no significant effects because of the low-pass ability of the actuator). The detection time is expressed in period numbers; thus, depending on the failure frequency, the time permissible for detection is varying. The Kalman filters have been widely used in fault detection and health monitoring of dynamic systems $[2,3]$. For nonlinear systems (and when it is desired to monitor some unknown parameters in addition to unknown states and outputs), the local filtering algorithms such as the extended Kalman filter (EKF), the Unscented filter (UF), or Divided Difference (DD) filter are usually used $[4,5]$. In these approaches, all conditional probability density functions of the state estimate are given by the first two moments, which induce local validity of the state estimates and, consequently, difficulty to improve the filter performance in generic case.

Contrasting its linear counterpart, the EKF in general is not an optimal estimator. In addition, if the initial estimate of the state is wrong, or if the process is modeled incorrectly, the filter may quickly diverge. Another problem with the EKF is that the estimated covariance matrix tends to underestimate the true covariance. The EKF typically works well only in the region where the 
first-order Taylor-series linearization adequately approximates the non-Gaussian probability density function. Moreover, the EKF assumes complete a priori knowledge of the process and measurement noise statistics. Whilst they are often assumed to be constant matrices, it is difficult to give accurate values for them. On the other hand, noise information plays an important role in the filtering algorithm: in particular, they determine the bandwidth of the filter and thus affect the convergence and stability.

So, when it comes to use the filter for fault detection and health monitoring, clearly a good trade-off between sensibility to faults and robustness with respect to normal environmental fluctuations is to be found. In other words, the noise covariance matrices are considered as design variables for fault detection and should be optimized in this context. Some works are available dealing with this problem from an "estimation" point of view (see [6-16] to mention a few). However, they do not take into account any fault detection sensitivity criteria. Namely, optimization of some estimation quality does not necessarily lead to an improvement of fault detection performance. The problem of tuning of parameters for fault detection performance optimization has been addressed in [17].

The objective of this paper is to propose a regular procedure for the noise covariance matrices adjustment in order to improve fault detection performance with application to the problem of OFC detection in flight control system and control surfaces in A380 [1, 18].

\section{FORMAL PROBLEM STATEMENT}

To be more specific, let consider a model of nonlinear system:

$$
\mathbf{x}_{k}=\mathbf{f}\left(\mathbf{x}_{k-1}, \mathbf{u}_{k-1}\right)+\mathbf{w}_{k-1} ; \quad \mathbf{y}_{k}=\mathbf{h}\left(\mathbf{x}_{k}\right)+\mathbf{v}_{k}
$$

where $\mathbf{x}_{k} \in R^{n}, \mathbf{u}_{k} \in R^{m}$, and $\mathbf{y}_{k} \in R^{p}$ are the system state, input, and output, respectively; and $\mathbf{w}_{k} \in R^{n}$ and $\mathbf{v}_{k} \in R^{p}$ are the process and observation noises, both assumed to be zero mean multivariate Gaussian noises with covariance $\mathbf{Q}_{k}$ and $\mathbf{R}_{k}$, respectively. The EKF equations have two parts, the prediction:

$$
\begin{aligned}
& \widehat{\mathbf{x}}_{k \mid k-1}=\mathbf{f}\left(\widehat{\mathbf{x}}_{k-1 \mid k-1}, \mathbf{u}_{k-1}\right) ; \\
& \mathbf{P}_{k \mid k-1}=\mathbf{F}_{k-1} \mathbf{P}_{k-1 \mid k-1} \mathbf{F}_{k-1}^{\mathrm{T}}+\mathbf{Q}_{k-1}
\end{aligned}
$$

and the update:

$$
\begin{gathered}
\mathbf{e}_{k}=\mathbf{y}_{k}-\mathbf{h}\left(\widehat{\mathbf{x}}_{k \mid k-1}\right) ; \quad \mathbf{S}_{k}=\mathbf{H}_{k} \mathbf{P}_{k \mid k-1} \mathbf{H}_{k}^{\mathrm{T}}+\mathbf{R}_{k} ; \\
\mathbf{K}_{k}=\mathbf{P}_{k \mid k-1} \mathbf{H}_{k}^{\mathrm{T}} \mathbf{S}_{k}^{-1}
\end{gathered}
$$




$$
\begin{aligned}
\widehat{\mathbf{x}}_{k \mid k} & =\widehat{\mathbf{x}}_{k \mid k-1}+\mathbf{K}_{k} \mathbf{e}_{k} \\
\mathbf{P}_{k \mid k} & =\left(\mathbf{I}-\mathbf{K}_{k} \mathbf{H}_{k}\right) \mathbf{P}_{k \mid k-1}
\end{aligned}
$$

where $\mathbf{F}_{k-1}=\partial \mathbf{f} / \partial \mathbf{x}_{\widehat{\mathbf{x}}_{k-1 \mid k-1}}$ and $\mathbf{u}_{k-1}$, and $\mathbf{H}_{k-1}=\partial \mathbf{h} / \partial \mathbf{x}_{\widehat{\mathbf{x}}_{k \mid k-1}}$. Equations (2) and (3) reproduce the predicted state $\widehat{\mathbf{x}}_{k \mid k-1}$ and the predicted estimate covariance $\mathbf{P}_{k \mid k-1}$. Innovations of the measurement residual $\mathbf{e}_{k}$ and the residual covariance $\mathbf{S}_{k}$ are computed in Eqs. (4). Equation (5) gives the optimal Kalman gain $\mathbf{K}_{k}$ and, finally, the state estimate $\widehat{\mathbf{x}}_{k \mid k}$ and the estimate covariance $\mathbf{P}_{k \mid k}$ are updated by Eqs. (6) and (7), respectively.

The matrices $\mathbf{Q}_{k}$ and $\mathbf{R}_{k}$ play an important role in the filtering algorithm: in particular, they determine the bandwidth of the filter and thus affect the convergence and stability. A large $\mathbf{Q}_{k}$ or small $\mathbf{R}_{k}$ means a wide bandwidth. In this condition, the filter can follow well the estimated quantities (state and/or parameters), but the filter must pay the price for it: by ignoring the "model information," the parameters become insensitive to the model; thus, they remain around their original values and reluctant to change. A small $\mathbf{Q}_{k}$ or large $\mathbf{R}_{k}$ represents a small bandwidth of the filter, which makes the parameters sensitive to the residuals. Although convergence speed is increased in this condition, the filter may not follow the estimated quantities.

Several regular procedures for the matrices $\mathbf{Q}_{k}$ and $\mathbf{R}_{k}$ adjustment are proposed in this work in order to improve fault detection performance in OFC (for brevity, only EKF is studied; however, the same algorithms can be applied for UF or DD filters with minor modifications).

The OFC problem is considered for the hydraulic actuator (see [1] for the background and the model details):

$$
\xi_{k+1}=\xi_{k}+K_{1}\left(u_{k}-\xi_{k}\right) \sqrt{\frac{\theta_{1}}{\Delta P_{\text {ref }}+K_{2}\left(u_{k}-\xi_{k}\right)^{2} \theta_{2}}}+w_{k} ; \quad z_{k}=\xi_{k}+v_{k}
$$

where $\xi_{k} \in R$ is the actuator rod position; $u_{k} \in R$ is the control signal; $z_{k} \in R$ is the available measurement output; $w_{k}$ and $v_{k}$ are the noises as before; $\Delta P_{\text {ref }}$ is the hydraulic differential pressure corresponding to the maximum rod speed; the values of parameters $\theta_{1}$ and $\theta_{2}$ are unknown, representing the aerodynamic forces applying on the control surface, hydraulic pressure delivered to the actuator (all of them for brevity of presentation are assumed to be unknown constant); and $K_{1}$ and $K_{2}$ are two known constants.

Two kinds of OFC are generally considered: "liquid" and "solid" failures. The first one is additive while the latter corresponds to oscillations replacing the original signal. The liquid failure is added to the normal signal: $u_{k}=u_{k}^{0}+f_{k}$, where $u_{k}^{0}$ is the nominal fault-free part (known by the fault detection system) and $f_{k}$ is the harmonic fault signal. The solid failure substitutes the normal signal: $u_{k}=f_{k}$. 
The OFC detection methodology must take into account the specificities of these two different cases. The most important real-time implementation constraints are [1]:

- the low computational complexity of a detection algorithm;

- the convergence must be proven; and

- a short time of convergence is required (due to the criticality of the failure detection).

In [19], the fault detection is carried out in two steps: residual generation and residual evaluation. First, a residual is generated by DD filter (under hypothesis of a fault-free command $u_{k}^{0}$ that is justified since the flight control law is also monitored by dedicated techniques). Second, the residual is decomposed in several spectral subbands. Counting oscillations in each subband of the filtered residual performs the OFC detection [1].

In this work, the attention will be focused on the residual generation only, to this end (8) is rewritten in the form (1) (including the unknown parameters):

$$
\begin{gathered}
\mathbf{x}_{k}=\left[\begin{array}{l}
\xi_{k} \\
\theta_{1} \\
\theta_{2}
\end{array}\right]=\left[\begin{array}{l}
x_{1, k} \\
x_{2, k} \\
x_{3, k}
\end{array}\right] ; \quad h\left(\mathbf{x}_{k}\right)=x_{1, k} ; \\
\mathbf{f}\left(\mathbf{x}_{k}, u_{k}\right) \\
=\left[x_{1, k}+K_{1}\left(u_{k}-x_{1, k}\right) \sqrt{\frac{x_{2, k}}{\Delta P_{\mathrm{ref}}+K_{2}\left(u_{k}-x_{1, k}\right)^{2} x_{3, k}}} x_{2, k} x_{3, k}\right]^{\mathrm{T}} .
\end{gathered}
$$

Then, the EKF is realized in accordance with (2)-(7) with $u_{k}=u_{k}^{0}$, initial conditions for the filter are chosen as $\widehat{\mathbf{x}}_{0}=\left[z_{o} \bar{\theta}_{1} \bar{\theta}_{2}\right]^{\mathrm{T}}$, where $\bar{\theta}_{1}$ and $\bar{\theta}_{2}$ are "the most probable" estimates on the parameters $\theta_{1}$ and $\theta_{2}$ values. The residual is defined as $r_{k}=y_{k}-h\left(\widehat{\mathbf{x}}_{k \mid k}\right)$ (in the designations above, as the difference between the measured actuator position $z_{k}$ and the estimated one $\left.\widehat{x}_{1, k}\right)$. If a fault appears, then $u_{k}=u_{k}^{0}+f_{k}$ or $u_{k}=f_{k}$ and the variable $\hat{x}_{1, k}$ (computed in accordance with the model ( 9$)$ for $u_{k}=u_{k}^{0}$ ) has to present a deviated behavior with respect to the measured $z_{k}$ that serves for OFC detection. The detection/estimation abilities of the EKF (2)-(7) in this case are seriously influenced by the matrices $\mathbf{Q}_{k}$ and $\mathbf{R}_{k}$ choice. Actually, after initial conditions, they are only incoming parameters of the EKF (2)-(7). Since the measurement noise statistics are relatively well identified comparing to the system model error, thus, for brevity, the matrix $\mathbf{R}_{k}$ is assumed to be known, and below, the problem of the matrix $\mathbf{Q}_{k}$ tuning is considered only. Finally, the same A380 flight dataset will be used through the paper for all simulations. 


\section{ESTIMATION BASED ADJUSTMENT OF COVARIANCE MATRICES}

In this section, three approaches are tested for adjustment of detection/ estimation abilities of the EKF. This comparison will motivate the main result of this paper that will be presented in the next section. The first one is the adaptive tuning of the matrix $\mathbf{Q}_{k}$ from $[8,11]$. The second approach is based on the Multimodel adaptive estimation (MMAE) approach from [12,20]. The third technique is the simplest one, but yet efficient, and it is based on iterative learning. To compare different algorithms, the estimation performance will be evaluated using the functional

$$
J=N^{-1} \sqrt{\sum_{k=1}^{N} \mathbf{e}_{k}^{\mathrm{T}} \mathbf{e}_{k}}
$$

where $N>0$ is the number of measured during flight tests samples.

\subsection{Adaptive Adjustment of $\mathrm{Q}_{k}$}

The following formula has been suggested in $[8,11]$ :

$$
\mathbf{Q}_{k}=\mathbf{Q}_{k-1}+\gamma\left(\mathbf{K}_{k} \mathbf{e}_{k} \mathbf{e}_{k}^{\mathrm{T}} \mathbf{K}_{k}^{\mathrm{T}}-\mathbf{P}_{k \mid k-1}+\mathbf{P}_{k-1 \mid k-1}-1 \mathbf{Q}_{k-1}\right)
$$

where $\gamma$ is the adaptation step (should be chosen in accordance with the trial and error method) and the rest variables are defined in (2)-(7). Realization

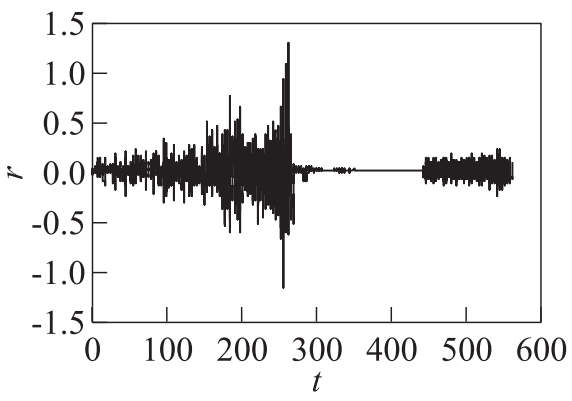

Figure 2 Residual for adaptive EKF of (10) does not require high computational power and its verification is very simple. The trial end error method shows that for an artificially generated data (for chosen $u_{k}^{0}$ and $w_{k}, v_{k}$, with given $\mathbf{Q}, \mathbf{R}$, for some initial conditions $\xi_{0}$ and $\bar{\theta}_{1}, \bar{\theta}_{2}$, using the model (8), the sequence $z_{k}$ is produced), there exists $\gamma>0$ such that $\mathbf{Q}_{k}$ in (10) converges asymptotically to a vicinity of $\mathbf{Q}$. However, for the real data, measured during A380 flight tests, the algorithm converges to different final values of $\mathbf{Q}_{k}$ depending on chosen initial conditions. A typical example of the residual $r_{k}$ is shown in Fig. $2(J=0.2495)$.

The algorithm (10) can also be tested for $\gamma<0$; in this case, the sequence $\mathbf{Q}_{k}$ is unstable, saturation and resetting to a predefined initial interval has to 
Table 1 Extended Kalman filter without adaptation for different scenarios

\begin{tabular}{cc}
\hline Minimal amplitude & $\begin{array}{c}\text { Detection } \\
\text { time, } \mathrm{s}\end{array}$ \\
\hline $0.31^{\circ}$ & 1.32 \\
$0.22^{\circ}$ & 1.56 \\
$0.21^{\circ}$ & 1.56 \\
$0.24^{\circ}$ & 1.56 \\
$0.24^{\circ}$ & 1.56 \\
$0.19^{\circ}$ & 1.56 \\
\hline
\end{tabular}

Table 2 Extended Kalman filter with adaptation for different scenarios

\begin{tabular}{cc}
\hline Minimal amplitude & $\begin{array}{c}\text { Detection } \\
\text { time, } \mathrm{s}\end{array}$ \\
\hline $0.28^{\circ}$ & 1.32 \\
$0.20^{\circ}$ & 1.56 \\
$0.19^{\circ}$ & 1.56 \\
$0.22^{\circ}$ & 1.56 \\
$0.21^{\circ}$ & 1.60 \\
$0.18^{\circ}$ & 1.56 \\
\hline
\end{tabular}

be used for all elements of the matrix $\mathbf{Q}_{k}$ to ensure boundedness. In this case, the adaptive EKF demonstrates rather good estimation performance on the real data. However, as in the case $\gamma>0$, it does not converge to a fixed value.

The following tests have been performed to evaluate the EKF fault detection ability $[1,18]$ (the minimal detectable amplitude of the fault and the fault detection time have been calculated for different $\mathbf{Q}_{k}$ ). First, a residual without failure is analyzed in order to compute a threshold of detection without false alarms. Second, once this threshold is determined, a fault is injected at a specific frequency to determine the magnitude of the detectable fault and detection time. The fault amplitude has been increased gradually to obtain a minimum detectable one for this frequency at selected instants of faults appearance. The second part is done for several frequencies and several instants of failure to cover all possible scenarios. The results of this procedure are presented in Tables 1 and 2. In Table 1, the minimal detectable amplitude and detection time are summarized for several scenarios of faults appearance on the frequency $2 \mathrm{~Hz}$ for conventional nonadaptive EKF (the liquid faults have been analyzed). In Table 2, the same results are given for adaptive EKF. Comparing these results, one may conclude that adaptation loop slightly decreases the minimal amplitude of detectable fault almost not influencing the detection time. However, the presence of adaptation decreases reliability of the detection, since the result of adjusting may depend on the fault structure.

\subsection{Multimodel Adaptive Estimations}

If there exist several admissible values for the matrix $\mathbf{Q}$, say $\mathbf{Q}^{1}, \ldots, \mathbf{Q}^{M}, M>0$, or the ranges of values for all elements of the matrix $\mathbf{Q}$ are known and the most probable their representations are $\mathbf{Q}^{i}, i=\overline{1, M}$, then MMAE approach can be applied $[12,20]$. This approach proposes to run all EKFs with different matrices $\mathbf{Q}^{i}, i=\overline{1, M}$, simultaneously, the likelihood of estimation performance for each particular EKF is calculated based on the corresponding estimation residual, the 


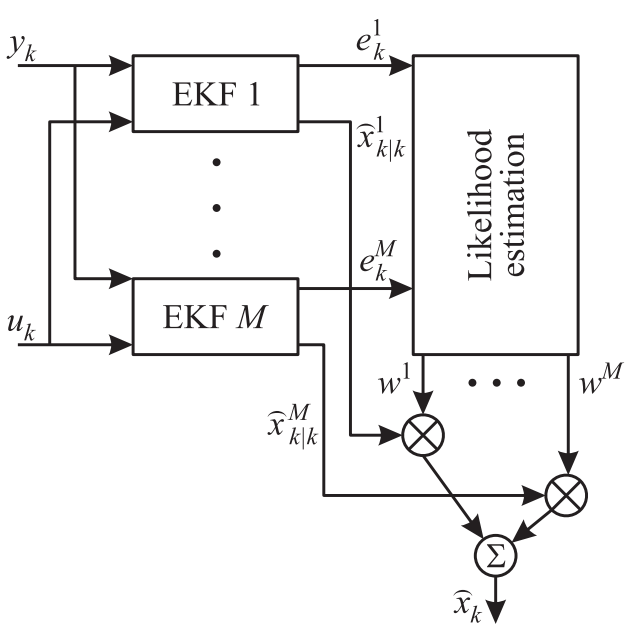

Figure 3 An MMAE scheme

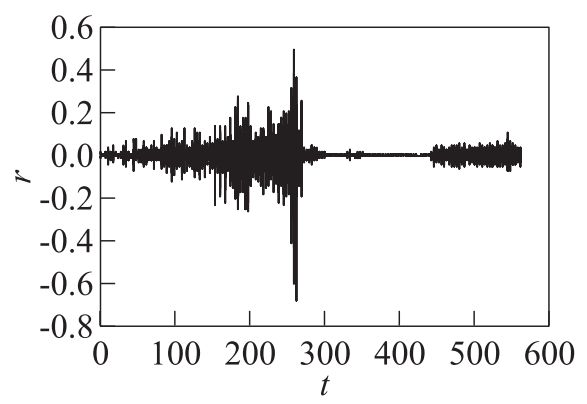

Figure 4 Residual for MMAE

highest weight is assigned to the EKF with the lowest output estimation error. The overall estimates $\mathbf{Q}$ and $\widehat{\mathbf{x}}_{k}$ are weighted averages of all elemental EKFs (Fig. 3).

The formula

$$
w^{i}=\frac{\widehat{\widetilde{w}}^{j}}{\sum_{j=1}^{M} \widehat{\widetilde{w}}^{j}}
$$

where

$$
\stackrel{\mathrm{w}}{w}^{j}=\frac{e^{-0.5 \mathbf{e}_{k}^{i \mathrm{~T}} \mathbf{S}_{k}^{i} \mathbf{e}_{k}^{i}}}{\sqrt{\operatorname{det}\left[2 \pi \mathbf{S}_{k}^{i}\right]}}, \quad i=\overline{1, M} ;
$$

$\mathbf{e}_{k}^{i}$ is the measurement residual; and $\mathbf{S}_{k}^{i}$ is the residual covariance (4) computed by the $i$ th EKF is used in [12] for likelihood derivation, then

$\widehat{\mathbf{x}}_{k \mid k}=\sum_{j=1}^{M} w^{i} \stackrel{\mathbf{x}}{k \mid k}_{i}^{i} ; \quad \mathbf{Q}_{k}=\sum_{i=1}^{M} w^{i} \mathbf{Q}_{k}^{i}$

where $\widehat{\mathbf{x}}_{k \mid k}$ and $\mathbf{Q}_{k}^{i}$ are the state estimates and the covariance estimates for $i$ th EKF, respectively.

The main drawback of this approach consists in its computational complexity ( $M$ EKFs have to be simulated in parallel). Its performance also critically depends on the choice of $\mathbf{Q}^{i}, i=\overline{1, M}$, if the grid $\mathbf{Q}^{i}$ has been chosen representative, then a good estimate $\mathbf{Q}_{k}$ can be obtained via MMAE. If the sequence $\mathbf{Q}^{i}, i=\overline{1, M}$, does not include a good approximate of the real covariance matrix, then the convergence properties are bad. An example of MMAE residual is shown in Fig. $4(J=0.0895)$.

\subsection{Iterative Learning}

The idea of this approach is described in the block scheme presented in Fig. 5. It starts with some initial guess value $\mathbf{Q}_{0}$ for which the estimation performance criteria $J_{0}$ is calculated. 


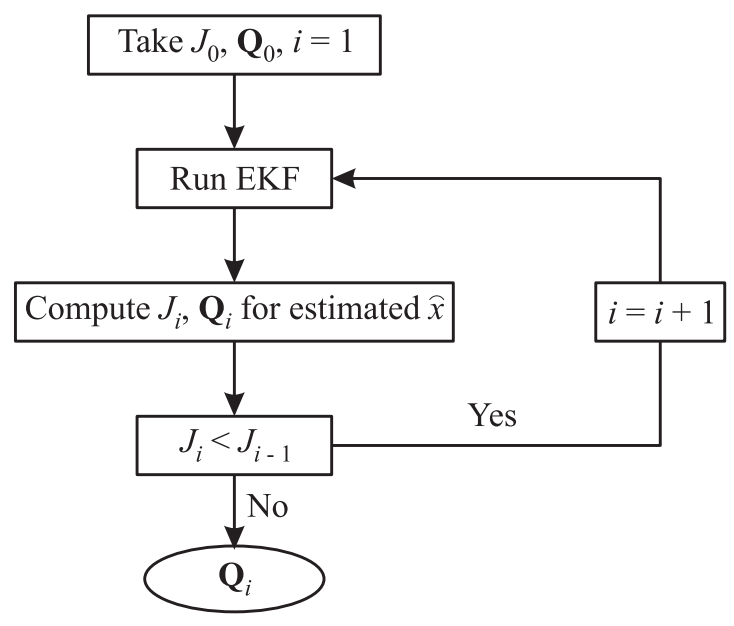

Figure 5 Scheme of iterative learning

After simulation, w can be estimated:

$$
\widehat{\mathbf{w}}_{k-1}=\widehat{\mathbf{x}}_{k \mid k}-\mathbf{f}\left(\widehat{\mathbf{x}}_{k-1 \mid k-1}, \mathbf{u}_{k-1}\right), \quad k=1, N
$$

then for the obtained vector $\widehat{\mathbf{w}}$, the next iteration matrix $\mathbf{Q}_{1}$ can be calculated, and after simulation, the value $J_{1}$ can be derived. If $J_{1}<J_{0}$, then the recurrent procedure has to be repeated in accordance with Fig. 5 . If $J_{i} \geq J_{i-1}$ (the estimation performance cannot be improved), then the procedure stops and the matrix from the last step $\mathbf{Q}_{i}$ is taken as an estimate of the true covariance matrix of the process noise.

An example of the residual obtained on the fourth iteration is shown in Fig. $6(J=0.0994)$. This procedure has low computational complexity, it can be applied offline and it demonstrates good improvement of optimization performance. Despite the value of the functional $J$ obtained here is slightly worse than one found by MMAE, this algorithm presents good compromise between computational complexity and estimation accuracy achieved.

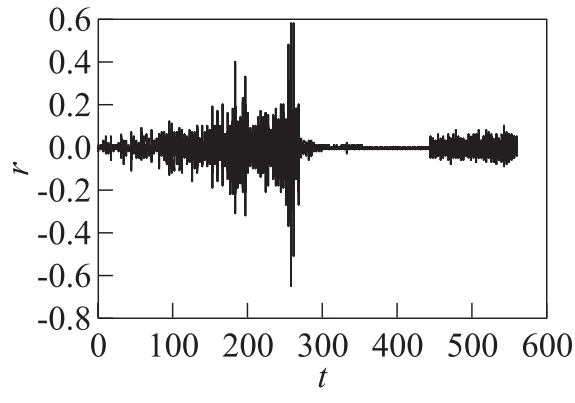

Figure 6 Residual for iterative learning 


\section{THE MAIN RESULT}

All approaches considered in the previous section are devoted to EKF estimation performance improvement via adjustment of the matrix $\mathbf{Q}_{k}$. Reformulation of them to the problem of fault detection meets several difficulties. The estimation performance can be directly characterized by the estimation error $\mathbf{e}_{k}$, while for the fault detection, it is necessary to take into account the estimation performance in the fault-free case, the sensitivity to faults, the robustness against perturbation, the false alarms avoiding, the time of detection, and so on. The list of these properties indicates that some of these characteristics can be evaluated a posteriori only, after the simulation run (e.g., estimation accuracy in the fault-free case and robustness against perturbations). Others depend on the fault models (like sensitivity to faults, false alarms, and time of detection). Thus, the dependence of them on $\mathbf{Q}_{k}$ is very complex and applications of gradient adaptive techniques, multiple models adaptation, or iterative learning, considered in the previous section, look questionable. Complex nonlinear dependence of fault detection performance on $\mathbf{Q}_{k}$ makes difficult an application of analytical optimization approaches.

The approach proposed in this work, to solve the problem of EKF fault detection performance improvement via the matrix $\mathbf{Q}_{k}$ tuning, consists in the following steps.

1. Choose the actuator model and the test dataset representing $u_{k}^{0}$ and $z_{k}, k=$ $\overline{1, N}, N>0$.

2. Choose the models of "the most probable" faults $f_{k}^{j}, j=\overline{1, K}, K>0$ with corresponding admissible times of detection $\tau^{j}, j=\overline{1, K}$.

3. Choose the grid of matrices $\mathbf{Q}^{i}, i=\overline{1, M}, M>0$, covering the range of possible values of the process noise covariance matrix.

4. Choose the performance criteria $I$ characterizing fault detection performance in the system.

5. Perform optimization of $I$ over grid $\mathbf{Q}^{i}, i=\overline{1, M}$, for chosen $f_{k}^{j}, \tau^{j}, j=\overline{1, K}$, and given $u_{k}^{0}, z_{k}, k=\overline{1, N}$.

The model of the actuator is given by (8), the parameters $K_{1}$ and $K_{2}$ depend on the actuator properties and the sampling rate. The test data set composed by the fault-free control $u_{k}^{0}$, examples of real measurements $z_{k}$, and the corresponding time instants $t_{k}$ is typically available after preliminary experiments. It has to represent the most typical and important operation modes of the actuator.

The models of faults for OFC case can be chosen as $f_{k}^{j}=A_{k}^{j} \sin \left(2 \pi \omega^{j} t_{k}\right)$, $k=\overline{1, N}, j=\overline{1, K}$, where $\omega^{j}$ lies in the range from 1 to $10 \mathrm{~Hz}$ (as it was explained above, the frequencies beyond $10 \mathrm{~Hz}$ are not considered because they are outside 
of the actuator dynamics bandwidth). The corresponding amplitudes $A_{k}^{j}$ depend on the frequencies $\omega^{j}$ and the current amplitude of the fault free control $u_{k}^{0}$ (for each frequency, several amplitudes can be chosen). The amplitudes $A_{k}^{j}=0$ could be chosen for some $k>0$ to simulate fault appearance and fading.

The grid of matrices $\mathbf{Q}^{i}, i=\overline{1, M}, M>0$, can be chosen using Monte Carlo method, or based on some a priori knowledge on the most representative exemplars from a given range.

The performance criteria for each $\mathbf{Q}^{i}, i=\overline{1, M}$, has to represent:

- the estimation performance in the fault-free case and the robustness against perturbation that can be defined as previously for $u_{k}=u_{k}^{0}$ by the functional $J_{0}^{i}=N^{-1} \sqrt{\sum_{k=1}^{N} \mathbf{e}_{k}^{\mathrm{T}} \mathbf{e}_{k}}$

- the sensitivity to faults can be characterized by the ratio $J_{j}^{i} / J_{0}^{i}, j=\overline{1, K}$, where $J_{j}^{i}=N^{-1} \sqrt{\sum_{k=1}^{N} \mathbf{e}_{k}^{\mathrm{T}} \mathbf{e}_{k}}$ is computed in the same way, but for $u_{k}$ $=u_{k}^{0}+f_{k}^{j}$ or $u_{k}=f_{k}^{j}$;

- the false alarms are avoided due to the fault detection algorithm design and thresholds adjusting $[1,18]$; and

- the (average) time of detection $T_{j}^{i}$ is also computed by the fault detection algorithm for each fault $f_{k}^{j}$.

The functional $J_{0}^{i}$ has to be minimized, the ratios $J_{j}^{i} / J_{0}^{i}, j=\overline{1, K}$, have to be maximized (increasing sensitivity), the detection times $T_{j}^{i}, j=\overline{1, K}$, have to satisfy obligatorily the constraint $T_{j}^{i} \leq \tau^{j}$. Consequently, the fault detection performance of EKF can be expressed as follows:

$$
I^{i}=\lambda J_{0}^{i}+(1-\lambda) K^{-1} \sum_{j=1}^{K}\left[\frac{J_{0}^{i}}{J_{j}^{i}}-\ln \left(T_{j}^{i} \leq \tau^{i}\right)\right], \quad i=\overline{1, M},
$$

where $0 \leq \lambda \leq 1$ is the weight constant regulating the influence of estimation or fault detection terms on the total value of the functional $I^{i}$. It is assumed that argument of the logarithm is 1 for $T_{j}^{i} \leq \tau^{j}$ (the condition is true), and the logarithm argument is 0 in the case $T_{j}^{i}>\tau^{j}$; thus, $\ln (0)=-\infty$ that penalizes $I^{i}$.

Having values $I^{i}$ of the performance functional on the grid $\mathbf{Q}^{i}, i=\overline{1, M}$, its optimization is straightforward:

$$
\mathbf{Q}^{*}=\mathbf{Q}^{i *}, \quad i^{*}=\arg \min _{i=1, M} I^{i} .
$$


Table 3 Values of the performance functionals $J_{0}^{i}$ and $J_{j}^{i}, i=\overline{1, M}, j=\overline{1, K}$

\begin{tabular}{ccccccccc}
\hline$i$ & $J_{0}$ & $J_{1}$ & $J_{2}$ & $J_{3}$ & $J_{4}$ & $J_{5}$ & $J_{6}$ & $J_{7}$ \\
\hline 1 & 0.1660 & 0.1705 & 0.17228 & 0.17727 & 0.17849 & 0.18807 & 0.21752 & 0.22022 \\
2 & 0.1525 & 0.1704 & 0.17222 & 0.17721 & 0.17843 & 0.18799 & 0.21740 & 0.22011 \\
3 & 0.1353 & 0.1703 & 0.17211 & 0.17709 & 0.17831 & 0.18786 & 0.21721 & 0.21993 \\
4 & 0.1366 & 0.1703 & 0.17212 & 0.17710 & 0.17832 & 0.18788 & 0.21728 & 0.22000 \\
5 & 0.1415 & 0.1703 & 0.17211 & 0.17709 & 0.17831 & 0.18786 & 0.21725 & 0.21997 \\
6 & 0.1550 & 0.1703 & 0.17207 & 0.17704 & 0.17826 & 0.18781 & 0.21716 & 0.21988 \\
7 & 0.1711 & 0.1702 & 0.17204 & 0.177 & 0.17822 & 0.18777 & 0.21711 & 0.21983 \\
8 & 0.1712 & 0.1702 & 0.17203 & 0.177 & 0.17822 & 0.18776 & 0.21711 & 0.21983 \\
9 & 0.1720 & 0.1702 & 0.17203 & 0.177 & 0.17822 & 0.18776 & 0.21710 & 0.21982 \\
\hline \hline$i$ & $J_{8}$ & $J_{9}$ & $J_{10}$ & $J_{11}$ & $J_{12}$ & $J_{13}$ & $J_{14}$ & $J_{15}$ \\
\hline 1 & 0.24000 & 0.29801 & 0.30208 & 0.32956 & 0.40519 & 0.40695 & 0.42855 & 0.49178 \\
2 & 0.23987 & 0.29781 & 0.30186 & 0.32928 & 0.40477 & 0.40651 & 0.42804 & 0.49127 \\
3 & 0.23968 & 0.29758 & 0.30162 & 0.32901 & 0.40446 & 0.40620 & 0.42773 & 0.49100 \\
4 & 0.23977 & 0.29774 & 0.30179 & 0.32923 & 0.40477 & 0.40651 & 0.42806 & 0.49134 \\
5 & 0.23973 & 0.29767 & 0.30171 & 0.32913 & 0.40461 & 0.40635 & 0.42788 & 0.49114 \\
6 & 0.23964 & 0.29754 & 0.30158 & 0.32897 & 0.40443 & 0.40617 & 0.42769 & 0.49097 \\
7 & 0.23958 & 0.29748 & 0.30152 & 0.32892 & 0.40439 & 0.40613 & 0.42767 & 0.49096 \\
8 & 0.23958 & 0.29748 & 0.30152 & 0.32892 & 0.40439 & 0.40612 & 0.42766 & 0.49094 \\
9 & 0.23957 & 0.29746 & 0.30150 & 0.32890 & 0.40435 & 0.40609 & 0.42762 & 0.49091 \\
\hline
\end{tabular}

The EKF with the covariance matrix $\mathbf{Q}^{*}$ ensures optimal fault detection performance taking in mind the chosen grid $\mathbf{Q}^{i}$, the fault models $f_{k}^{j}$, and the given test dataset. The proposed procedure has simple computer implementation and can be performed offline for given actuator.

This procedure has been again applied to the same A380 dataset. A sample logarithmic grid with $M=9$ has been generated with the uniform range of diagonal elements of the matrices $\mathbf{Q}^{i}, i=\overline{1, M}$, from 0.01 to 1 . Next, for $K=15$, several models of "liquid" faults have been generated with the range of amplitudes from 0.2 to 0.6 and frequencies from 1 to $7 \mathrm{~Hz} ; \tau^{j}=3 / \omega^{j}, j=\overline{1, K}$. The instants of the faults appearance and fading have been assigned ( 3 times per test). The performance functional (11) has been chosen. The calculated values of the functionals $J_{0}^{i}$ and $J_{j}^{i}, i=\overline{1, M}, j=\overline{1, K}$, are presented in Table 3 .

During simulation, the condition $T_{j}^{i} \leq \tau^{j}$ have been satisfied for all matrices $\mathbf{Q}^{i}, i=\overline{1, M}$, and faults $j=\overline{1, K}$. Choosing different values of the weighting coefficient $\lambda$, one can obtain different optimal matrices $\mathbf{Q}^{*}$. In Table 4 , the values of $I^{i}, i=\overline{1, M}$, are presented for $\lambda=0.9$ and 0.1 , with $i^{*}=3$ and 4 , correspondingly. Therefore, if the accuracy of the system estimation in the faultfree case is less important, the matrix $\mathbf{Q}^{3}$ provides better fault detection performance. If the estimation accuracy is imperative, then the EKF with the 
matrix $\mathbf{Q}^{4}$ application is more reasonable. This conclusion easily follows by the $J_{0}$ column of the Table 3 analysis, $J_{0}^{4}<J_{0}^{3}$. The considered application of the proposed approach serves for the illustration purpose. For real application, the numbers of covariance matrices $\mathbf{M}$ and faults $\mathbf{K}$ have to be chosen bigger.

\section{CONCLUDING REMARKS}

The problem of the EKF fault detection performance optimization by the covariance matrices $\mathbf{Q}_{k}$ tuning is considered for OFC detection in A380. Several estimation based approaches existent in the literature for the matrix adjustment are considered and compared: adaptive tuning, multiple-model adaptive estimation, and iterative learning. Novel global optimization procedure for the fault detection abilities of EKF is formulized and applied to the problem of oscillatory failures detection. Computer simulation confirms efficiency of the proposed approach.

\section{ACKNOWLEDGMENTS}

The authors would like to thank Philippe Goupil (Airbus, Toulouse, Flight Control System Department) for his valuable comments.

\section{REFERENCES}

1. Goupil, P. 2010. Oscillatory failure case detection in the A380 electrical flight control system by analytical redundancy. Control Eng. Practice. doi:10.1016/j.conengprac.2009.04.003.

2. Beard, R. V. 1971. Failure accommodation in linear system through selfreorganization. Ph.D. Thesis. Massachussets Institute of Technology.

3. Jones, H. L. 1973. Failure detection in linear systems. Ph.D. Thesis. Massachussets Institute of Technology.

4. Chen, J., and R. J. Patton. 1999. Robust model-based fault diagnosis for dynamic systems. Norwell, MA: Kluwer Academic Publs.

5. Blanke, M., M. Kinnaert, M. Lunze, and M. Staroswiecki. 2003. Diagnosis and fault tolerant control. New York: Springer.

6. Nørgård, P., N. Poulsen, and O. Ravn. 2000. New developments in state estimation for nonlinear systems. Automatica 36(11):1627-38. 
7. Leu, G., and R. Baratti. 2000. An extended Kalman filtering approach with a criterion to set its tuning parameters; application to a catalytic reactor. Computers Chem. Eng. 23(11-12):1839-49.

8. Busse, F. D., and J. P. How. 2002. Demonstration of adaptive extended Kalman filter for low Earth orbit estimation using DGPS. Institute of Navigation GPS Meeting.

9. Powell, T. D. 2002. Automated tuning of an extended Kalman filter using the downhill simplex algorithm. J. Guidance Control Dyn. 25(5):901-8.

10. Crassidis, J. L., and F. L. Markley. 2003. Unscented filtering for spacecraft attitude estimation. J. Guidance Control Dyn. 26:536-42.

11. Lee, D. 2005. Nonlinear bayesian filtering with applications to estimation and navigation. Ph.D. Thesis. Texas A\&M University.

12. Wyffels, K. L. 2007. Development of a ground truth simulator and application of a generalized multiple-model adaptive estimation approach to tune a state estimation filter. Ph.D. Thesis. NY: Rochester Institute of Technology.

13. Åkesson, B. M., J. B. Jørgensen, N. K. Poulsen, and S. B. Jørgensen. 2008. A generalized auto covariance least-squares method for Kalman filter tuning. J. Process Control 18(7-8):769-79.

14. Alsuwaidan, B. 2008. Convergence properties of autocorrelation-based generalized multiple-model adaptive estimation. AIAA Guidance, Navigation and Control Conference and Exhibit Proceedings. Honolulu, Hawaii.

15. Wiberg, D., H. H. Sewon, Y. J. Su, L. Johnson, and H. S. Kyo. 2008. A fix-up for the EKF parameter estimator. 17th IFAC World Congress Proceedings. Seoul, Korea. 6502-7.

16. Subrahmanya, N., and Y.C. Shin. 2009. Adaptive divided difference filtering for simultaneous state and parameter estimation. Automatica 45(7):1686-93.

17. Marzat, J., E. Walter, H. Piet-Lahanier, and F. Damongeot. 2010. Automatic tuning via Kriging-based optimization of methods for fault detection and isolation. IEEE Conference on Control and Fault-Tolerant Systems (SysTol'10) Proceedings. Nice. 505-10.

18. Alcorta-Garcia, E., A. Zolghadri, P. Goupil, L. Lavigne, and P. Simon. 2009. Nonlinear observer-based OFC detection for A380 aircraft. Safe Process'09 Proceedings. Barcelona, Spain.

19. Zolghadri, A., C. Goetz, B. Bergeon, and X. Denoise. 1998. Integrity monitoring of flight parameters using analytical redundancy. UKACC International Conference on Control (CONTROL'98) Proceedings. U.K. 1534-39.

20. Hanlonand, P.D., and P.S. Maybeck. 2000. Multiple-model adaptive estimation using a residual correlation Kalman FilterBank. IEEE Trans. Aerospace Electronic Syst. AES-36(2):393-406. 